\title{
THE SIMULTANEOUS APPROXIMATION ORDER BY NEURAL NETWORKS WITH A SQUASHING FUNCTION
}

\author{
NAHMWOO НАHм
}

\begin{abstract}
In this paper, we study the simultaneous approximation to functions in $C^{m}[0,1]$ by neural networks with a squashing function and the complexity related to the simultaneous approximation using a Bernstein polynomial and the modulus of continuity. Our proofs are constructive.
\end{abstract}

\section{Introduction}

It is well known that an approximation by neural networks is based on superpositions of a transfer function. A feedforward neural network with a transfer function $\sigma: \mathbb{R} \rightarrow \mathbb{R}$ and $n$ neurons in the hidden layer is given by

$$
\sum_{i=1}^{n} a_{i} \sigma\left(b_{i} x+c_{i}\right)
$$

where $b_{i}$ 's are weights and $c_{i}$ 's are thresholds. Many researchers have proved the density results $[1,5,7]$ and the complexity results $[11,12,13]$ by neural networks. Especially, simultaneous approximations of functions and their derivatives have been studied in recent years by many authors $[2,4,6,8,9]$ since they have many applications in the fields of science and engineering. Most results in $[2,4,6,9]$ investigated the density results of simultaneous approximations by neural networks. $\mathrm{Li}$ and $\mathrm{Xu}[8]$ showed the simultaneous approximation order by neural networks with a trigonometric function. The goal of this paper is to obtain the simultaneous approximation order of functions in $C^{m}[0,1]$ and their derivatives by neural networks with a sigmoidal function. A sigmoidal function is a function $\sigma: \mathbb{R} \rightarrow \mathbb{R}$ such that

$$
\lim _{x \rightarrow-\infty} \sigma(x)=0 \text { and } \quad \lim _{x \rightarrow \infty} \sigma(x)=1 .
$$

Received September 8, 2008.

2000 Mathematics Subject Classification. Primary 41A10, 41A29, 65D15.

Key words and phrases. simultaneous approximation, Bernstein polynomial, squashing function, neural network. 
The following functions are some sigmoidal functions.

$$
\begin{array}{ll}
\text { Heaviside function : } & \sigma_{h}(x)= \begin{cases}1 & \text { if } x \geq 0 \\
0 & \text { if } x<0 .\end{cases} \\
\text { Squashing function : } & \sigma_{c}(x)=\frac{1}{1+c e^{-x}}, \quad c \text { is a positive constant. }
\end{array}
$$

It is well known that continuous functions on a compact subset of $\mathbb{R}$ can be approximated well by neural networks with the Heaviside function. But, the Heaviside function is not differentiable. Therefore, in this paper, we choose a squashing function $\sigma(x)=1 /\left(1+e^{-x}\right)$ as a transfer function of neural networks. In addition, neural networks with a squashing function are important due to historical reasons [14] as a suitable model for the response characteristics of a biological neuron.

\section{Preliminaries}

Throughout the paper, $m$ denotes a fixed positive integer, $c, c_{1}, c_{2}, c_{3}, \ldots$ denote positive constants and their values may be different at different occurrences.

In order to get the main result, we use a Bernstein polynomial. By the binomial theorem and the properties of a Bernstein polynomial [10], we have the following result.

Lemma 2.1. Let $n \in \mathbb{N}$ be given. Then, for any integer $r$ with $0 \leq r \leq n$ and $x \in[0,1]$, the followings hold.

(a) $\sum_{k=0}^{n-r}\left(\begin{array}{c}n-r \\ k\end{array}\right) x^{k}(1-x)^{n-r-k}=1$.

(b) $\sum_{k=0}^{n-r}\left(\begin{array}{c}n-r \\ k\end{array}\right) k x^{k}(1-x)^{n-r-k}=(n-r) x$.

(c) $\sum_{k=0}^{n-r}\left(\begin{array}{c}n-r \\ k\end{array}\right) k^{2} x^{k}(1-x)^{n-r-k}=(n-r) x((n-r-1) x+1)$.

The following lemma can be directly obtained from Lemma 2.1.

Lemma 2.2. Let $n \in \mathbb{N}$ be given. Then, for any integer $r$ with $0 \leq r \leq n$, we have

$$
\left\|T_{n, r}\right\|_{\infty,[0,1]} \leq \frac{n-r}{4}
$$

where $T_{n, r}(x)=\sum_{k=0}^{n-r}(k-(n-r) x)^{2}\left(\begin{array}{c}n-r \\ k\end{array}\right) x^{k}(1-x)^{n-r-k}$ for $x \in[0,1]$. 
Proof. By Lemma 2.1, we have

(1) $T_{n, r}(x)=\sum_{k=0}^{n-r}(k-(n-r) x)^{2}\left(\begin{array}{c}n-r \\ k\end{array}\right) x^{k}(1-x)^{n-r-k}$

$$
\begin{aligned}
& =\sum_{k=0}^{n-r}\left\{k^{2}-2(n-r) k x+(n-r)^{2} x^{2}\right\}\left(\begin{array}{c}
n-r \\
k
\end{array}\right) x^{k}(1-x)^{n-r-k} \\
& =(n-r) x\{(n-r-1) x+1-(n-r) x\} \\
& =(n-r)\left(x-x^{2}\right) .
\end{aligned}
$$

Therefore $\left|T_{n, r}(x)\right| \leq(n-r) / 4$ for any $x \in[0,1]$.

For $f \in C^{m}[0,1]$ and $\delta>0$, we define the modulus of continuity of $f^{(r)}$ for $r \in \mathbb{Z}$ with $0 \leq r \leq m$ as follows.

$$
\omega\left(f^{(r)}, \delta\right):=\sup \left\{\left|f^{(r)}(x)-f^{(r)}(y)\right|:|x-y|<\delta\right\},
$$

where $x, y \in[0,1]$. Note that $\omega\left(f^{(r)}, \delta\right)$ satisfies the followings.

(a) $\omega\left(f^{(r)}, \delta\right)$ is a non-decreasing function.

(b) $\omega\left(f^{(r)}, \alpha \delta\right) \leq(\alpha+1) \omega\left(f^{(r)}, \delta\right)$ for a positive real number $\alpha$.

(c) $\omega\left(f^{(r)}, \alpha+\beta\right) \leq \omega\left(f^{(r)}, \alpha\right)+\omega\left(f^{(r)}, \beta\right)$ for positive real numbers $\alpha, \beta$.

For any nonnegative integer $n$, the difference of a function $f$ with a step $h$ is defined by

$$
\Delta_{h}^{n} f(x):=\Delta_{h}\left(\Delta_{h}^{n-1} f(x)\right),
$$

where $\Delta_{h}^{0} f(x):=f(x)$ and $\Delta_{h} f(x):=\Delta_{h}^{1} f(x)=f(x+h)-f(x)$.

\section{Main results}

In this section, first, we obtain the simultaneous approximation order of functions in $C^{m}[0,1]$ by Bernstein polynomials. Then we show that Bernstein polynomials are simultaneously approximated by neural networks with a squashing function using the properties of a squashing function.

Theorem 3.1. Let $f \in C^{m}[0,1]$ and $n \in \mathbb{N}$ with $m<n$. Then, for any integer $r$ with $0 \leq r \leq m$, we have

$$
\left\|f^{(r)}-B_{n}^{(r)}(f)\right\|_{\infty,[0,1]} \leq c_{1} \omega\left(f^{(r)}, \frac{1}{\sqrt{n-r}}\right)+\frac{c_{2}}{n},
$$

where $c_{1}$ and $c_{2}$ are positive constants independent of $n$.

Proof. For $r=0$, it is proved in [10] that

$$
\left\|f-B_{n}(f)\right\|_{\infty,[0,1]} \leq c \omega\left(f, \frac{1}{\sqrt{n}}\right),
$$


where $c$ is a positive constant independent of $n$. Note that

$$
\text { (3) } \begin{aligned}
B_{n}^{\prime}(f, x) & =\sum_{k=0}^{n} f\left(\frac{k}{n}\right)\left(\begin{array}{l}
n \\
k
\end{array}\right)\left[k x^{k-1}(1-x)^{n-k}-(n-k) x^{k}(1-x)^{n-1-k}\right] \\
& =n \sum_{k=0}^{n-1}\left[f\left(\frac{k+1}{n}\right)-f\left(\frac{k}{n}\right)\right]\left(\begin{array}{c}
n-1 \\
k
\end{array}\right) x^{k}(1-x)^{n-1-k} \\
& =n \sum_{k=0}^{n-1} \Delta_{1 / n} f\left(\frac{k}{n}\right)\left(\begin{array}{c}
n-1 \\
k
\end{array}\right) x^{k}(1-x)^{n-1-k} .
\end{aligned}
$$

By differentiating $B_{n}^{\prime}(f, x)$ and simplifying the computation, we get (4)

$$
\begin{aligned}
& B_{n}^{\prime \prime}(f, x) \\
= & n(n-1) \sum_{k=0}^{n-2}\left[f\left(\frac{k+2}{n}\right)-2 f\left(\frac{k+1}{n}\right)+f\left(\frac{k}{n}\right)\right]\left(\begin{array}{c}
n-2 \\
k
\end{array}\right) x^{k}(1-x)^{n-2-k} \\
= & n(n-1) \sum_{k=0}^{n-2} \Delta_{1 / n}^{2} f\left(\frac{k}{n}\right)\left(\begin{array}{c}
n-2 \\
k
\end{array}\right) x^{k}(1-x)^{n-2-k} .
\end{aligned}
$$

Inductively, we can easily obtain

(5) $B_{n}^{(r)}(f, x)=n(n-1) \cdots(n-r+1) \sum_{k=0}^{n-r} \Delta_{1 / n}^{r} f\left(\frac{k}{n}\right)\left(\begin{array}{c}n-r \\ k\end{array}\right) x^{k}(1-x)^{n-r-k}$

for $r$ with $1 \leq r \leq m$. According to the properties of the divided difference [3], there exists $\theta_{k, r} \in[k / n,(k+r) / n]$ such that

$$
\Delta_{1 / n}^{r} f\left(\frac{k}{n}\right)=\left(\frac{1}{n}\right)^{r} f^{(r)}\left(\theta_{k, r}\right) .
$$

Thus, by (6), (5) can be rewritten as

$$
B_{n}^{(r)}(f, x)=\prod_{j=0}^{r-1}\left(1-\frac{j}{n}\right) \sum_{k=0}^{n-r} f^{(r)}\left(\theta_{k, r}\right)\left(\begin{array}{c}
n-r \\
k
\end{array}\right) x^{k}(1-x)^{n-r-k}
$$

for $r$ with $1 \leq r \leq m$. Now we estimate the simultaneous approximation order by a Bernstein polynomial. For $x \in[0,1]$, we have

$$
\begin{aligned}
& \left|f^{(r)}(x)-B_{n}^{(r)}(f, x)\right| \\
\leq & \prod_{j=0}^{r-1}\left(1-\frac{j}{n}\right) \sum_{k=0}^{n-r}\left|f^{(r)}(x)-f^{(r)}\left(\theta_{k, r}\right)\right|\left(\begin{array}{c}
n-r \\
k
\end{array}\right) x^{k}(1-x)^{n-r-k} \\
& +\left[1-\prod_{j=0}^{r-1}\left(1-\frac{j}{n}\right)\right]\left|f^{(r)}(x)\right| .
\end{aligned}
$$


We compute the first part of the right side of (8). Note that

(9)

$$
\begin{aligned}
& \prod_{j=0}^{r-1}\left(1-\frac{j}{n}\right) \sum_{k=0}^{n-r}\left|f^{(r)}(x)-f^{(r)}\left(\theta_{k, r}\right)\right|\left(\begin{array}{c}
n-r \\
k
\end{array}\right) x^{k}(1-x)^{n-r-k} \\
\leq & \sum_{k=0}^{n-r}\left|f^{(r)}(x)-f^{(r)}\left(\theta_{k, r}\right)\right|\left(\begin{array}{c}
n-r \\
k
\end{array}\right) x^{k}(1-x)^{n-r-k} \\
\leq & \sum_{k=0}^{n-r}\left|f^{(r)}(x)-f^{(r)}\left(\frac{k}{n-r}\right)\right|\left(\begin{array}{c}
n-r \\
k
\end{array}\right) x^{k}(1-x)^{n-r-k} \\
& +\sum_{k=0}^{n-r}\left|f^{(r)}\left(\frac{k}{n-r}\right)-f^{(r)}\left(\theta_{k, r}\right)\right|\left(\begin{array}{c}
n-r \\
k
\end{array}\right) x^{k}(1-x)^{n-r-k} .
\end{aligned}
$$

Now we compute an upper bound of

$$
\sum_{k=0}^{n-r}\left|f^{(r)}(x)-f^{(r)}\left(\frac{k}{n-r}\right)\right|\left(\begin{array}{c}
n-r \\
k
\end{array}\right) x^{k}(1-x)^{n-r-k}
$$

in (9). Let $\delta>0$ be given. For $x_{0}, y_{0} \in[0,1]$, we set $\alpha:=\alpha\left(x_{0}, y_{0}, \delta\right)$ an integer $\left[\left|y_{0}-x_{0}\right| / \delta\right]$, where [.] is the Gauss function. For $i=0,1,2, \ldots, \alpha+1$, we define $q_{i}=x_{0}+\left(\left(y_{0}-x_{0}\right) /(\alpha+1)\right) i$. Then

$$
\left|f^{(r)}\left(x_{0}\right)-f^{(r)}\left(y_{0}\right)\right| \leq \sum_{i=0}^{\alpha}\left|f^{(r)}\left(q_{i+1}\right)-f^{(r)}\left(q_{i}\right)\right| \leq(\alpha+1) \omega\left(f^{(r)}, \delta\right)
$$

for $r$ with $1 \leq r \leq m$. By Lemma 2.2 and (10), we have

$$
\begin{aligned}
& \sum_{k=0}^{n-r}\left|f^{(r)}(x)-f^{(r)}\left(\frac{k}{n-r}\right)\right|\left(\begin{array}{c}
n-r \\
k
\end{array}\right) x^{k}(1-x)^{n-r-k} \\
\leq & \omega\left(f^{(r)}, \delta\right) \sum_{k=0}^{n-r}\left[\alpha\left(x, \frac{k}{n-r}, \delta\right)+1\right]\left(\begin{array}{c}
n-r \\
k
\end{array}\right) x^{k}(1-x)^{n-r-k} \\
\leq & \omega\left(f^{(r)}, \delta\right)\left[\sum_{k=0}^{n-r} \alpha\left(x, \frac{k}{n-r}, \delta\right)\left(\begin{array}{c}
n-r \\
k
\end{array}\right) x^{k}(1-x)^{n-r-k}+1\right] \\
\leq & \omega\left(f^{(r)}, \delta\right)\left[\frac{1}{\delta^{2}} \sum_{k=0}^{n-r}\left(\frac{k}{n-r}-x\right)^{2}\left(\begin{array}{c}
n-r \\
k
\end{array}\right) x^{k}(1-x)^{n-r-k}+1\right] \\
\leq & \omega\left(f^{(r)}, \delta\right)\left(\frac{1}{4(n-r) \delta^{2}}+1\right) .
\end{aligned}
$$

If we choose $\delta=1 / \sqrt{n-r}$, then, by (11), we have

$$
\sum_{k=0}^{n-r}\left|f^{(r)}(x)-f^{(r)}\left(\frac{k}{n-r}\right)\right|\left(\begin{array}{c}
n-r \\
k
\end{array}\right) x^{k}(1-x)^{n-r-k} \leq \frac{5}{4} \omega\left(f^{(r)}, \frac{1}{\sqrt{n-r}}\right) .
$$


Finally, we compute an upper bound of

$$
\sum_{k=0}^{n-r}\left|f^{(r)}\left(\frac{k}{n-r}\right)-f^{(r)}\left(\theta_{k, r}\right)\right|\left(\begin{array}{c}
n-r \\
k
\end{array}\right) x^{k}(1-x)^{n-r-k}
$$

in (9). Note that $k / n \leq k /(n-r) \leq(k+r) / n$ for $r$ with $1 \leq r \leq m<n$ and any integer $k$ with $0 \leq k \leq n-r$. Since $\theta_{k, r} \in[k / n,(k+r) / n]$, we have

$$
\begin{aligned}
& \sum_{k=0}^{n-r}\left|f^{(r)}\left(\frac{k}{n-r}\right)-f^{(r)}\left(\theta_{k, r}\right)\right|\left(\begin{array}{c}
n-r \\
k
\end{array}\right) x^{k}(1-x)^{n-r-k} \\
\leq & \sum_{k=0}^{n-r} \omega\left(f^{(r)}, \frac{r}{n}\right)\left(\begin{array}{c}
n-r \\
k
\end{array}\right) x^{k}(1-x)^{n-r-k} .
\end{aligned}
$$

By the properties of the modulus of continuity,

$$
\omega\left(f^{(r)}, \frac{r}{n}\right) \leq(r+1) \omega\left(f^{(r)}, \frac{1}{n}\right) \leq(r+1) \omega\left(f^{(r)}, \frac{1}{\sqrt{n-r}}\right) .
$$

From (12), (13) and (14), we have an upper bound of (9) such that

$$
\begin{aligned}
& \prod_{j=0}^{r-1}\left(1-\frac{j}{n}\right) \sum_{k=0}^{n-r}\left|f^{(r)}(x)-f^{(r)}\left(\theta_{k, r}\right)\right|\left(\begin{array}{c}
n-r \\
k
\end{array}\right) x^{k}(1-x)^{n-r-k} \\
\leq & \frac{5}{4} \omega\left(f^{(r)}, \frac{1}{\sqrt{n-r}}\right)+(r+1) \omega\left(f^{(r)}, \frac{1}{\sqrt{n-r}}\right) \\
:= & c_{1} \omega\left(f^{(r)}, \frac{1}{\sqrt{n-r}}\right),
\end{aligned}
$$

where $c_{1}$ is a constant independent of $n$.

Now we compute the second part of the right side of (8). Since

$$
\begin{aligned}
1-\prod_{j=0}^{r-1}\left(1-\frac{j}{n}\right)= & {\left[1-\left(1-\frac{1}{n}\right)\right]+\left[\left(1-\frac{1}{n}\right)-\left(1-\frac{1}{n}\right)\left(1-\frac{2}{n}\right)\right]+\cdots } \\
& +\left[\left(1-\frac{1}{n}\right) \cdots\left(1-\frac{r-2}{n}\right)-\left(1-\frac{1}{n}\right) \cdots\left(1-\frac{r-1}{n}\right)\right] \\
= & \sum_{i=1}^{r-1} \frac{i}{n} \prod_{j=0}^{i-1}\left(1-\frac{j}{n}\right) \\
\leq & \sum_{i=1}^{r-1} \frac{i}{n}=\frac{r(r-1)}{2 n}
\end{aligned}
$$

we have

$$
\left[1-\prod_{j=0}^{r-1}\left(1-\frac{j}{n}\right)\right]\left|f^{(r)}(x)\right| \leq \frac{r(r-1)}{2 n} M_{r}:=\frac{c_{2}}{n}
$$


where $M_{r}:=\left\|f^{(r)}\right\|_{\infty,[0,1]}$ for $r$ with $1 \leq r \leq m$ and $c_{2}$ is a constant independent of $n$. From (2), (15) and (17), we get

$$
\left\|f^{(r)}-B_{n}^{(r)}(f)\right\|_{\infty,[0,1]} \leq c_{1} \omega\left(f^{(r)}, \frac{1}{\sqrt{n-r}}\right)+\frac{c_{2}}{n}
$$

for $r$ with $0 \leq r \leq m$, where $c_{1}$ and $c_{2}$ are positive constants independent of $n$.

If $n$ is sufficiently large in Theorem 3.1, we are able to obtain the improved simultaneous approximation order as follows.

Theorem 3.2. Let $f \in C^{m}[0,1]$ and $n \in \mathbb{N}$ with $2 m<n$. Then, for any integer $r$ with $0 \leq r \leq m$, we have

$$
\left\|f^{(r)}-B_{n}^{(r)}(f)\right\|_{\infty,[0,1]} \leq c_{1} \omega\left(f^{(r)}, \frac{1}{\sqrt{n}}\right)+\frac{c_{2}}{n},
$$

where $c_{1}$ and $c_{2}$ are positive constants independent of $n$.

Proof. Since $2 m<n$, we have $n / 2=n-n / 2<n-m \leq n-r$ and so

$$
\omega\left(f^{(r)}, \frac{1}{\sqrt{n-r}}\right)<\omega\left(f^{(r)}, \frac{\sqrt{2}}{\sqrt{n}}\right) \leq(\sqrt{2}+1) \omega\left(f^{(r)}, \frac{1}{\sqrt{n}}\right) .
$$

By Theorem 3.1 and (19), we complete the proof.

Note that a squashing function $\sigma(x)=1 /\left(1+e^{-x}\right)$ is a nonlinear, monotone increasing and differentiable sigmoidal function. In addition, it has a nonvanishing point in $[0,1]$ by the following two lemmas.

Lemma 3.3. Suppose that $R_{n}(\sigma):=R_{n}(\sigma, x)$ denotes a polynomial of degree $\leq n$ with respect to $\sigma$ for $n \in \mathbb{N}$. Then

$$
\sigma^{(n)}(x)=\sigma(x)(1-\sigma(x)) R_{n-1}(\sigma)
$$

for any $n \in \mathbb{N}$.

Proof. We prove it by the mathematical induction.

For $n=1$, it is clear that $\sigma^{\prime}(x)=\sigma(x)(1-\sigma(x))$.

Assume that (20) is true for $n=k$. That is, $\sigma^{(k)}(x)=\sigma(x)(1-\sigma(x)) R_{k-1}(\sigma)$.

Then, for $n=k+1$, we have

$$
\begin{aligned}
& \sigma^{(k+1)}(x) \\
= & \sigma^{\prime}(x)\left[(1-\sigma(x)) R_{k-1}(\sigma)-\sigma(x) R_{k-1}(\sigma)+\sigma(x)(1-\sigma(x)) R_{k-1}^{\prime}(\sigma)\right] \\
= & \sigma(x)(1-\sigma(x)) R_{k}(\sigma),
\end{aligned}
$$

since $(1-\sigma(x)) R_{k-1}(\sigma), \sigma(x) R_{k-1}(\sigma)$ and $\sigma(x)(1-\sigma(x)) R_{k-1}^{\prime}(\sigma)$ are polynomials of degree $\leq k$ with respect to $\sigma$. This completes the proof.

Lemma 3.4. Let $\sigma(x)=1 /\left(1+e^{-x}\right)$. Then there exists $x_{0} \in[0,1]$ such that $\sigma^{(n)}\left(x_{0}\right) \neq 0$ for any $n \in \mathbb{N}$. 
Proof. Note that $\sigma(x) \neq 0$ and $1-\sigma(x) \neq 0$ for all $x \in[0,1]$. Since $\sigma(x)$ is monotone increasing on $\mathbb{R}, R_{k}(\sigma)=0$ has at most $k$ roots in $[0,1]$. Thus $\sigma^{(k+1)}(x)=0$ has at most $k$ roots by $(21)$. So $\left\{x \in[0,1]: \bigcup_{n=1}^{\infty} \sigma^{(n)}(x)=0\right\}$ is countable and hence there exists $x_{0} \in[0,1]$ such that $x_{0} \in[0,1]-\{x \in[0,1]$ : $\left.\bigcup_{n=1}^{\infty} \sigma^{(n)}(x)=0\right\}$.

Similarly, we can easily show that any squashing function $\sigma_{c}(x)=1 /(1+$ $c e^{-x}$ ) with a positive constant $c$ also has a non-vanishing point. Using Lemma 3.3, Lemma 3.4 and the divided difference, we now approximate monomials simultaneously by neural networks with a squashing function.

Lemma 3.5. Let $\sigma$ be a squashing function and $k \in \mathbb{N} \cup\{0\}$. If $b \in[0,1]$ such that $\sigma^{(j)}(b) \neq 0$ for any $j \in \mathbb{N}$ and $h>0$, there exists a neural network

$$
N_{k, h}(\sigma, x)=\frac{1}{h^{k} \sigma^{(k)}(b)} \sum_{j=0}^{k}\left(\begin{array}{l}
k \\
j
\end{array}\right)(-1)^{k-j} \sigma(h j x+b)
$$

such that

$$
\left\|\left(x^{k}\right)^{(r)}-N_{k, h}^{(r)}\right\|_{\infty,[0,1]}=\mathcal{O}(h)
$$

for any integer $r$ with $0 \leq r \leq m$.

Proof. For $r=0, N_{k, h}(\sigma, x)$ represents the divided difference for $x^{k}$ and so

$$
\left\|x^{k}-N_{k, h}\right\|_{\infty,[0,1]}=\mathcal{O}(h)
$$

holds. In order to compute the derivatives of $N_{k, h}(\sigma, x)$, we define

$$
[p]_{q}=\prod_{i=0}^{q-1}(p-i)
$$

for $p, q \in \mathbb{N}$. For $p, s \in \mathbb{N}$, we choose $a_{q, s} \in \mathbb{R}$ for $q=1,2, \ldots, s$ so that

$$
p^{s}=\sum_{q=1}^{s} a_{q, s}[p]_{q}
$$

From (24), we get

$$
\frac{[j]_{q}}{[k]_{q}}\left(\begin{array}{c}
k \\
j
\end{array}\right)=\frac{j(j-1) \cdots(j-(q-1))}{k(k-1) \cdots(k-(q-1))} \frac{k !}{j !(k-j) !}=\left(\begin{array}{c}
k-q \\
j-q
\end{array}\right)
$$


for $q, j, k \in \mathbb{N}$ with $q \leq j \leq k$. Using (24) and (25), we compute the $r$-th derivative of $N_{k, h}(x)$ for $r$ with $1 \leq r \leq m$.

$(26)$

$$
\begin{aligned}
& N_{k, h}^{(r)}(\sigma, x) \\
= & \frac{1}{h^{k} \sigma^{(k)}(b)} \sum_{j=0}^{k}\left(\begin{array}{c}
k \\
j
\end{array}\right)(-1)^{k-j} \sigma^{(r)}(h j x+b) h^{r} j^{r} \\
= & \frac{1}{h^{k-r} \sigma^{(k)}(b)} \sum_{j=1}^{k}\left(\begin{array}{c}
k \\
j
\end{array}\right)(-1)^{k-j} \sum_{q=1}^{r} a_{q, r}[j]_{q} \sigma^{(r)}(h j x+b) \\
= & \frac{1}{h^{k-r} \sigma^{(k)}(b)} \sum_{q=1}^{r} a_{q, r}[k]_{q} \sum_{j=q}^{k}\left(\begin{array}{c}
k-q \\
j-q
\end{array}\right)(-1)^{k-j} \sigma^{(r)}(h j x+b) \\
= & \frac{1}{h^{k-r} \sigma^{(k)}(b)} \sum_{q=1}^{r} a_{q, r}[k]_{q} \sum_{i=0}^{k-q}\left(\begin{array}{c}
k-q \\
i
\end{array}\right)(-1)^{k-i-q} \sigma^{(r)}(h i x+h q x+b) .
\end{aligned}
$$

By Taylor's theorem for an integer $p$ with $p>k-r$, we have

$$
\begin{aligned}
\sigma^{(r)}(h i x+h q x+b)= & \sigma^{(r)}(h i x+b)+\sum_{l=1}^{p-1} \frac{\sigma^{(r+l)}(h i x+b)}{l !}(h q x)^{l} \\
& +\frac{\sigma^{(r+p)}\left(\xi_{i, q}\right)}{p !}(h q x)^{p},
\end{aligned}
$$

where $\xi_{i, q}$ is a point between $h i x+b$ and $h i x+h q x+b$. Note that

$$
\begin{aligned}
& \frac{1}{h^{k-r} \sigma^{(k)}(b)} \sum_{q=1}^{r} a_{q, r}[k]_{q} \sum_{i=0}^{k-q}\left(\begin{array}{c}
k-q \\
i
\end{array}\right)(-1)^{k-i-q} \sigma^{(r)}(h i x+b) \\
= & \sum_{q=1}^{r} a_{q, r}[k]_{q} h^{r-q} N_{k-q, h}\left(\sigma^{(r)}, x\right) \\
= & a_{r, r}[k]_{r} N_{k-r, h}\left(\sigma^{(r)}, x\right)+\sum_{q=1}^{r-1} a_{q, r}[k]_{q} h^{r-q} N_{k-q, h}\left(\sigma^{(r)}, x\right) \\
= & {[k]_{r} N_{k-r, h}\left(\sigma^{(r)}, x\right)+\mathcal{O}(h), }
\end{aligned}
$$

since $a_{r, r}=1$ by comparing the leading coefficients in (24). Since $l+r-q \geq 1$, we have

$$
\frac{1}{h^{k-r} \sigma^{(k)}(b)} \sum_{q=1}^{r} a_{q, r}[k]_{q} \sum_{i=0}^{k-q}\left(\begin{array}{c}
k-q \\
i
\end{array}\right)(-1)^{k-i-q}\left[\sum_{l=1}^{p-1} \frac{\sigma^{(r+l)}(h i x+b)}{l !}(h q x)^{l}\right]
$$




$$
\begin{aligned}
& =\sum_{l=1}^{p-1} \sum_{q=1}^{r} a_{q, r}[k]_{q} h^{l+r-q}\left[\frac{1}{h^{k-q} \sigma^{(k)}(b)} \sum_{i=0}^{k-q}(-1)^{k-i-q} \sigma^{(r+l)}(h i x+b)\right] \\
& =\sum_{l=1}^{p-1} \sum_{q=1}^{r} a_{q, r}[k]_{q} h^{l+r-q}\left[x^{k-q}+\mathcal{O}(h)\right] \\
& =\mathcal{O}(h) .
\end{aligned}
$$

Moreover, since $\sigma^{(r+p)}\left(\xi_{i, q}\right)$ is bounded for $\xi_{i, q} \in[0,1]$ and $p-k+r \geq 1$, we have

(30)

$$
\frac{1}{h^{k-r} \sigma^{(k)}(b)} \sum_{q=1}^{r} a_{q, r}[k]_{q} \sum_{i=0}^{k-q}\left(\begin{array}{c}
k-q \\
i
\end{array}\right)(-1)^{k-i-q} \frac{\sigma^{(r+p)}\left(\xi_{i, q}\right)}{p !}(h q x)^{p}=\mathcal{O}(h) .
$$

From (28), (29) and (30), we have

$$
N_{k, h}^{(r)}(\sigma, x)=[k]_{r} N_{k-r, h}\left(\sigma^{(r)}, x\right)+\mathcal{O}(h) .
$$

Therefore

(32) $\left\|\left(x^{k}\right)^{(r)}-N_{k, h}^{(r)}\right\|_{\infty,[0,1]}=\left\|[k]_{r} x^{k-r}-[k]_{r} N_{k-r, h}\left(\sigma^{(r)}, x\right)\right\|_{\infty,[0,1]}=\mathcal{O}(h)$ for $r$ with $1 \leq r \leq m$. By (22) and (32), we complete the proof.

The next theorem follows from Lemma 3.5 immediately.

Theorem 3.6. Let $\epsilon>0$ be given and let $\sigma$ be a squashing function. If $b \in[0,1]$ such that $\sigma^{(j)}(b) \neq 0$ for any $j \in \mathbb{N}$ and $P_{n}(x)=\sum_{k=0}^{n} a_{k} x^{k}$ for $n \in \mathbb{N}$, there exists a neural network

$N_{n}(\sigma, x):=\sum_{k=0}^{n} a_{k} N_{k, h}(\sigma, x)=\sum_{k=0}^{n} a_{k}\left[\frac{1}{h^{k} \sigma^{(k)}(b)} \sum_{j=0}^{k}(-1)^{k-j}\left(\begin{array}{l}k \\ j\end{array}\right) \sigma(h j x+b)\right]$

such that

$$
\left\|P_{n}^{(r)}-N_{n}^{(r)}(\sigma)\right\|_{\infty,[0,1]}<\epsilon
$$

for sufficiently small $h>0$ and any integer $r$ with $0 \leq r \leq m$.

By combining Theorem 3.2 and Theorem 3.6, we get the following theorem that is the main result of this paper.

Theorem 3.7. Let $f \in C^{m}[0,1]$ and $n \in \mathbb{N}$ with $2 m<n$. If $\sigma$ is a squashing function and $b \in[0,1]$ such that $\sigma^{(j)}(b) \neq 0$ for any $j \in \mathbb{N}$, there exists a neural network

$$
N_{n}(\sigma, x)=\sum_{k=0}^{n} a_{k}\left[\frac{1}{h^{k} \sigma^{(k)}(b)} \sum_{j=0}^{k}(-1)^{k-j}\left(\begin{array}{c}
k \\
j
\end{array}\right) \sigma(h j x+b)\right]
$$

such that

$$
\left\|f^{(r)}-N_{n}^{(r)}(\sigma)\right\|_{\infty,[0,1]} \leq c_{1} \omega\left(f^{(r)}, \frac{1}{\sqrt{n}}\right)+\frac{c_{2}}{n}
$$


where $a_{k}$ 's are the coefficients of the Bernstein polynomial with resect to $f$, and constants $c_{1}$ and $c_{2}$ are independent of $n$ for sufficiently small $h>0$ and any integer $r$ with $0 \leq r \leq m$.

Proof. By Theorem 3.2, we have

$$
\left\|f^{(r)}-B_{n}^{(r)}(f)\right\|_{\infty,[0,1]} \leq c_{1} \omega\left(f^{(r)}, \frac{1}{\sqrt{n}}\right)+\frac{c_{2}}{n}
$$

for $r$ with $0 \leq r \leq m$, where $c_{1}$ and $c_{2}$ are positive constants independent of $n$. For a given $\epsilon>0$, we get, by Theorem 3.6,

$$
\left\|B_{n}^{(r)}(f)-N_{n}^{(r)}(\sigma)\right\|_{\infty,[0,1]}<\epsilon
$$

for sufficiently small $h>0$ and $r$ with $0 \leq r \leq m$. Therefore

$$
\begin{aligned}
& \left\|f^{(r)}-N_{n}^{(r)}(\sigma)\right\|_{\infty,[0,1]} \\
\leq & \left\|f^{(r)}-B_{n}^{(r)}(f)\right\|_{\infty,[0,1]}+\left\|B_{n}^{(r)}(f)-N_{n}^{(r)}(\sigma)\right\|_{\infty,[0,1]} \\
\leq & c_{1} \omega\left(f^{(r)}, \frac{1}{\sqrt{n}}\right)+\frac{c_{2}}{n}+\epsilon .
\end{aligned}
$$

Since $\epsilon>0$ is arbitrarily small, we complete the proof.

\section{References}

[1] R. M. Burton and H. G. Dehling, Universal approximation in p-mean by neural networks, Neural Networks 11 (1998), 661-667.

[2] P. Cardaliaguet and G. Euvrard, Approximation of a function and its derivative with a neural network, Neural Networks 5 (1992), 207-220.

[3] R. A. DeVore and G. G. Lorentz, Constructive Approximation, Springer Verlag, Heidelberg, 1993.

[4] A. R. Gallant and H. White, On learning the derivatives of an unknown mapping with multilayer feedforward networks, Lett. Math. Phys. 5 (1992), 129-138.

[5] B. Gao and Y. Xu, Univariant approximation by superpositions of a sigmoidal function, J. Math. Anal. Appl. 178 (1993), no. 1, 221-226.

[6] Y. Ito, Simultaneous $L_{p}$-approximations of polynomials and dervatives on the whole space, Arti. Neural Networks Conf. (1999), 587-592.

[7] G. Lewicki and G. Marino, Approximation of functions of finite variation by superpositions of a sigmoidal function, Appl. Math. Lett. 17 (2004), no. 10, 1147-1152.

[8] F. Li and $\mathrm{Z}$. Xu, The essential order of simultaneous approximation for neural networks, Appl. Math. Comput. 194 (2007), no. 1, 120-127.

[9] X. Li, Simultaneous approximation of a multivariate functions and their derivatives by neural networks with one hidden layer, Neurocomputing 12 (1996), 327-343.

[10] G. G. Lorentz, Bernstein Polynomials, Chelsea, Engelwood Cliffs, 1986.

[11] B. Malakooti and Y. Q. Zhou, Approximation polynomial functions by feedforward artificial neural networks : capacity analysis and design, Appl. Math. and Comp. 90 (1998), $27-51$.

[12] M. V. Medvedeva, On sigmoidal functions, Moscow Univ. Math. Bull. 53 (1998), no. 1, $16-19$.

[13] H. N. Mhaskar and N. Hahm, Neural networks for functional approximation and system identification, Neural Computation 9 (1997), no. 1, 143-159.

[14] D. E. Rumelhart, G. E. Hinton, and R. J. Williams, Parallel Distributed Processing : explorations in the microstructure of cognition, MIT Press, Massachusetts, 1986. 
Department of Mathematics

UNIVERSITY OF INCHEON

INCHEON 402-749, KOREA

E-mail address: nhahm@incheon.ac.kr 